\title{
Endoscopic Staging and Treatment of Early Gastric Cancer
}

\author{
Angel Ferrández ${ }^{1,2}$, Sonia Mostacero ${ }^{3}$, Angel Lanas ${ }^{1,2,4}$ \\ ${ }^{1}$ Service of Digestive Diseases, Hospital Clínico Lozano Blesa, Zaragoza, Spain; ${ }^{2}$ The Centro de Investigación Biomédica en Red, \\ Barcelona, Spain; ${ }^{3}$ Fundación Hospital de Calahorra, La Rioja, Spain; ${ }^{4}$ Universidad de Zaragoza, Zaragoza, Spain. \\ Email: angel.ferrandez@telefonica.net
}

Received November $19^{\text {th }}, 2012$; revised December $20^{\text {th }}, 2012$; accepted December $29^{\text {th }}, 2012$

\begin{abstract}
Gastric cancer is the most common cancer worldwide and it is often diagnosed in an advanced stage. In countries where screening endoscopy is performed widely, early detection is possible. In fact, early gastric cancer incidence is increasing during the last years worldwide and screening could be a major factor in such increase. In the past, the standard treatment of gastric cancer was surgical resection; however, the endoscopic treatment has increased due to advances in the instruments available and clinician experience. In fact, endoscopic resection has become one of the greatest advances in EGC treatment. It is the standard treatment in most of the cases because early gastric cancer is associated with a low rate of lymph node metastasis and a high survival rate. Endoscopic Mucosal Resection and more recently Endoscopic Submucosal Dissection are the two main developed procedures. Endoscopic Submucosal Dissection achieves a higher rate of en-bloc resection, complete resection, curative resection and lower local recurrence compared with Endoscopic Mucosal Resection group. The disadvantages associated with Endoscopic Submucosal Dissection, such as higher perforation rates and longer procedure time, will probably improve as the endoscopists experience increases and new endoscopic tools are developed. The aim of this paper is to review the management of EGC with a special focus on endoscopic detection, staging, therapy, surveillance, and prevention.
\end{abstract}

Keywords: Early Gastric Cancer; Endoscopic Mucosa Resection; Endoscopic Submucosal Dissection; Endoscopic Ultrasound

\section{Introduction}

Early gastric cancer (EGC) is defined as a cancer that does not invade beyond the submucosa regardless of lymph node involvement. The proportion of EGC at diagnosis is increasing due to the use of mass screening endoscopy and it represents up to $40 \%-60 \%$ of all gastric cancer in Japan. Low risk of lymph node metastases, and over $90 \% 5$-year survival rate have made this tumor an ideal candidate for endoscopic treatment [1].

Over the past 20 years novel procedures for endoscopic resection have been developed. They include mucosal and more recently submucosal resection.

In Japan, the first description of endoscopic polypectomy as a treatment for pedunculated or semipedunculated EGC was presented in 1974 and in 1984 Tada and colleagues reported a revolutionary Endoscopic Mucosa Resection (EMR) technique called strip biopsy [2]. A few years later, another EMR technique was developed also in Japan, using a standard endoscopic needle knife with local injection of a hypertonic saline epinephrine [3]. The step forward was a new endoscopic resection tech- nique that included the submucosal cut of lesions with special endoscopic knives. It was developed in the late 1990s and it has recently been classified as Endoscopic Submucosal Dissection (ESD) to distinguish it from conventional EMR. The first ESD procedure was performed with an insulation-tipped (IT) diathermic knife, developed in 1996 at the National Cancer Center Hospital, Tokyo [4].

The endoscopic procedures together with the development of new staging technology allow this cancer to be treated with a similar efficacy, less invasively and in a more cost-effective manner compared to open surgery [5].

\section{Diagnosis of Early Gastric Cancer}

Early diagnosis represents the most important factor to decrease gastric cancer mortality. Once a lesion is detected, efforts should be made to describe it accurately. The macroscopic classification of early and advanced GC was proposed by the Japanese Gastric Cancer Association [6], which has been internationally accepted, and is 
shown in Table 1.

Recent development of new imaging techniques improve accuracy and detection of EGC that include narrow band imaging (NBI), chromoendoscopy and confocal laser endomicroscopy (CLE).

Nowadays, NBI is considered the preferred endoscopic technique in EGC diagnosis over chromoendoscopy or CLE. Magnification chromoendoscopy is a safe and inexpensive technique but it has a limited applicability due to the long duration of the procedure. CLE is a sophisticated technology that allows real-time microscopic analysis of mucosal and submucosal structures. In fact, magnifying NBI, in conjunction with conventional white light improves lesion identification of both techniques alone. It identifies small, depressed gastric mucosal cancers with $96.6 \%$ accuracy, $95.0 \%$ sensitivity, and $96.8 \%$ specificity [7].

However its price and the fact that it is very timeconsuming makes this technique unpractical for extended use.

A head to head comparison study of NBI, magnification chromoendoscopy and white-light endoscopy (WLE) by Zhang et al. [8] showed that NBI was superior to conventional endoscopy in the diagnosis of EGC and precancerous gastric lesions, and can be used for screening early malignancies of the stomach. In fact, the accuracy of WLE in the diagnosis of EGC was $68.9 \%$ compared to $93.6 \%$ for NBI and $91.3 \%$ for conventional magnifying

Table 1. Endoscopic classification of gastric cancer.

\begin{tabular}{l}
\hline Early gastric cancer (type 0): Superficial protruding or \\
non-protruding lesions \\
\hline Protruding \\
Pedunculated 0-Ip \\
Sessile: 0-Is \\
Non-protruding and non-excavated \\
Slightly elevated 0-IIa \\
Completely flat 0-IIb \\
Slightly depressed o-IIc \\
Elevated and depressed types 0IIc+IIa or 0IIa+IIc \\
Excavated \\
Ulcer 0-III \\
Excavated and depressed types 0-IIc+III or 0-III+IIc \\
\hline Advanced gastric cancer \\
Type 1: Protruding carcinoma, attached on a wide base \\
Type 2: Ulcerated carcinoma with sharp and raised margins \\
Type 3: Ulcerated carcinoma without definite limits \\
Type 4: Non. ulcerated, diffusely infiltrating carcinoma \\
Type 5: Unclassificable advanced carcinoma \\
\hline
\end{tabular}

endoscopy. Moreover, NBI and chromoendoscopy were significantly superior to magnifying conventional endoscopy for image resolution including morphology, pit pattern, and blood capillary form $(\mathrm{p}<0.01)$, and magnifying NBI was significantly superior to magnifying chromoendoscopy for blood capillary form $(\mathrm{p}<0.01)$. A recent validation for a classification of gastric lesions according to NBI findings has been published and it is shown in Table 2 [9].

\section{Staging Early Gastric Cancer}

Clinical baseline stage provides useful information for the development of an initial treatment strategy. Approximately $50 \%$ of patients will present with advanced disease at diagnosis and have a poor outcome. Clinical staging has improved with the availability of diagnostic modalities that include Endoscopic Ultrasound (EUS), Computered Tomography (CT), Multi Detector row Computered Tomography (MDCT), Positron Emission Tomography combined with CT (PET/CT) and Magnetic Resonance Imaging (MRI).

Radical surgery represents the main treatment option with curative intent in gastric cancer and new approaches (mainly endoscopic) are gaining importance in the therapeutic management of these patients. According to the TNM classification system (Table 3), T stage is the most determinant factor for an appropriate treatment, as well as the most important predictor of survival in patients with gastric cancer [10].

The agreement between pre-operative TNM staging by imaging tools and post-operative staging by pathology is not perfect and may affect treatment decisions. Operator dependence and heterogeneity of data may account for the variations in staging performance [11]. That is why the novel therapeutic strategies will require reliable staging procedures in order to choose the most appropriate treatment for each patient.

Table 2. Classification of precancerous lesions based on NBI findings.

\begin{tabular}{cccc}
\hline Pattern & Endoscopic findings & Pathology & $\begin{array}{c}\text { Accuracy } \% \\
(95 \% \mathrm{CI})\end{array}$ \\
\hline A & $\begin{array}{c}\text { Regular vessels with } \\
\text { circular mucosa }\end{array}$ & Normal & $83(75-90)$ \\
B & Tubulovillous mucosa & $\begin{array}{c}\text { Intestinal } \\
\text { metaplasia }\end{array}$ & $84 \%(77-90)$ \\
\hline Light blue crest & $\begin{array}{c}\text { Intestinal } \\
\text { metaplasia }\end{array}$ & $80(73-86)$ \\
& $\begin{array}{c}\text { Irregular vessels and } \\
\text { mucosa }\end{array}$ & Dysplasia & $95(90-99)$ \\
& Variable vascular \\
density & H. pylori & $70(59-80)$ \\
\hline
\end{tabular}

Adapted from Pimentel-Nunes, P., et al. [9]. 
Table 3. Endoscopic classification of gastric cancer.

\begin{tabular}{|c|c|}
\hline \multicolumn{2}{|c|}{$\mathrm{T}$ category definitions } \\
\hline$T x$ & Primary tumor cannot be assessed \\
\hline T0 & No evidence of primary tumor \\
\hline Tis & $\begin{array}{l}\text { Carcinoma in situ: intraepithelial tumor without } \\
\text { invasion of the lamina propria }\end{array}$ \\
\hline $\mathrm{T} 1$ & $\begin{array}{l}\text { Tumor invades lamina propria, muscularis mucosae or } \\
\text { submucosa }\end{array}$ \\
\hline T1a & Tumor invades lamina propria or muscularis mucosae \\
\hline $\mathrm{T} 1 \mathrm{~b}$ & Tumor invades submucosa \\
\hline $\mathrm{T} 2$ & Tumor invades muscularispropria \\
\hline $\mathrm{T} 3$ & $\begin{array}{l}\text { Tumor penetrates subserosal connective tissue without } \\
\text { invasion of visceral peritoneum or adjacent strictures. } \\
\text { T3 tumors also include those extending into the } \\
\text { gastrocolic or gastrohepatic ligaments, or onto the } \\
\text { greater or lesser omentum, without perforation of the } \\
\text { visceral peritoneum covering these structures }\end{array}$ \\
\hline $\mathrm{T} 4$ & $\begin{array}{l}\text { Tumor invades serosa (visceral peritoneum) or adjacent } \\
\text { structures }\end{array}$ \\
\hline $\mathrm{T} 4 \mathrm{a}$ & Tumor invades serosa (visceral peritoneum) \\
\hline $\mathrm{T} 4 \mathrm{~b}$ & $\begin{array}{l}\text { Tumor invades adjacent structures such as spleen, } \\
\text { transverse colon, liver, diaphragm, pancreas, abdominal } \\
\text { wall, adrenal gland, kidney, small intestine and } \\
\text { retroperitoneum }\end{array}$ \\
\hline \multicolumn{2}{|c|}{$\mathrm{N}$ category definitions } \\
\hline $\mathrm{Nx}$ & Regional lymph nodes cannot be assessed \\
\hline N0 & No regional lymph node metastases \\
\hline N1 & Metastasis in 1 to 2 regional lymph nodes \\
\hline $\mathrm{N} 2$ & Metastasis in 3 to 6 regional lymph nodes \\
\hline N3 & Metastasis in 7 or more regional lymph nodes \\
\hline
\end{tabular}

\subsection{Endoscopic Ultrasound}

Endoscopic Ultrasound has been used since 1980 and has become one of the most important tools to examine the biliary and gastrointestinal tracts. EUS combines endoscopy and high-frequency ultrasound allowing a complete visualization of the gastric wall and the adjacent structures such as the peri-gastric lymph nodes. Normal gastric walls appear as five-layered structure, whereas a gastric carcinoma is identified by disruption, thickening, and irregularity of such layers [12].

There are some contradictory reports on the role of EUs in the diagnosis of EGC. However, most of the studies suggest that EUs is the most accurate technique for T staging of gastric cancer. Such accuracy is due to its ability to define the layers of the gastric wall and its ability to diagnose vertical cancer invasion depth of the tumor. EUS may be technically challenging because the optical control of such a large device for small lesions is not always feasible and the resolution can be inadequate in superficial lesions. Moreover, clinical and pathological characteristics of the tumor as size, location, concomitant ulceration and histologically type, may affect the diagnostic performance of EUS [13].

Endoscopic Ultrasound evaluation presents a high per- formance rate in differentiating early and intermediate (T1 - T2) from advanced (T3 - T4) primary gastric tumors. However, its accuracy for individual $\mathrm{T}$ categories (T1 - T4) is lower [14]. The average EUS performance for differentiation of $\mathrm{T} 1 \mathrm{~m}$ from $\mathrm{T} 1 \mathrm{sm}$ lesions, which would allow selecting patients for endoscopic treatment, is not informative for clinical practice. Respect from $\mathrm{N}$ status, this meta-analysis shows that the ability of EUS to distinguish between positive and negative node involvement is not satisfactory. However it increases the probability of being staged as node+ from $55 \%$ to $84 \%$, and it lowers the same probability to $31 \%$ when there is node involvement.

This problem seems to have been resolved with the recent introduction of high frequency thin endoscopic ultrasound probes that allow endoscopists to perform target scanning of very small gastric cancer lesion under endoscopic control [15]. It is very plausible that technological improvements may lead to better EUS performance rates and to optimization gastric cancer staging.

\subsection{Multi-Detector Row Computered Tomography}

MDCT is a relatively new promising technology for gastric imaging that allows $3 \mathrm{D}$ reconstruction of the stomach. New MDCT devices allow an enhanced visualization of the mucosal layer of the stomach [16]. However, he results of MDCT in local tumor staging of EGC might be insufficient. In fact, previous studies show a low effectiveness of CT for T staging of gastric cancer of $43 \%$ with an overall accuracy of $82 \%$. Although MDCT allows visualization of distant regions, the exposure of the patient to radiation is a major disadvantage that favors the use of EUS in EGC.

\subsection{Magnetic Resonance Imaging}

MRI performance is similar to MDTC but its use in gastric cancer staging is very restricted, mainly due to its limited availability, low-quality imaging secondary to motion artifacts, the lack of stable contrasts, and a high procedure-related cost.

There are very few studies comparing EUS, MDCT, and MRI. Bhandari et al. [17] made a direct comparison between MDCT and EUS in 63 patients with 67 gastric cancers and no major differences between the two methods were found. However, a recent metanalyses demonstrated that pre-operative $\mathrm{T}$ staging MRI scans had better performance accuracy than CT or MDCT [11]. Results are shown in Table 4.

\section{Treatment}

Endoscopic resection is the standard treatment for EGD 
Table 4. Performance of different techniques in gastric cancer diagnoses.

\begin{tabular}{cccccc}
\hline & & 3D-MDCT & CT & MRI & EUS \\
\hline \multirow{2}{*}{ T-stage } & Accuracy & $80.4 \pm 2.7$ & $71.5 \pm 2.7$ & $82.9 \pm 3.7$ & 87.5 \\
& Sensitivity & & 69.1 & & $86(81-90)$ \\
& Specificity & & 94.4 & & $91(89-93)$ \\
N-stage & Accuracy & $67.1 \pm 2.6$ & $66.1 \pm 2.1$ & $53.4 \pm 5.9$ & 79.1 \\
& Sensitivity & & $77.2 \pm 2.6$ & $85.3 \pm 4.7$ & $69(63-74)$ \\
& Specificity & & $78.3 \pm 2.5$ & $75.0 \pm 9.3$ & $84(81-88)$ \\
\hline
\end{tabular}

Data modified from References [11,14,17].

in Japan and its gaining acceptance in Western countries in the last years.

Endoscopic resection is similar in efficacy to surgery, less invasive, more cost-effective, improves patient quality of life and allows accurate histological staging of the cancer, which is necessary to decide what patients will need additional treatment [18].

The two major endoscopic techniques for EGC are endoscopic mucosal resection [EMR] and endoscopic submucosal dissection [ESD].

\subsection{Endoscopic Mucosal Resection}

This technique was introduced in Japan in the 1980s and it is and advanced form of snare polypectomy that allows complete resection of small tumors. Endoscopic mucosal resection (EMR) is widely accepted as a minimally invasive treatment for early gastric cancer.

It allows local curative and minimally invasive treatment in situations that otherwise require radical surgery, low cost, patient tolerance, and better patient quality of life after the operation. EMR is indicated when the risk of lymph node metastasis is minimal, and the tumor can be removed en bloc. In the guidelines by the Japanese Gastric Cancer Association differentiated mucosal cancers measuring less than $2 \mathrm{~cm}$ best fit the above criteria. The main advantages versus conventional polypectomy are, first, that allows an en-bloc resection of the specimen that is necessary to provide precise histological diagnosis and second, that it can also reduces the recurrence rate [19].

With the development of endoscopic technique and improvement of the devices, more and more new techniques based on EMR have been used in clinic practice, such as EMR-precut, EMR-cap, and EMR-ligation. However, conventional EMR is limited by the size of the specimen allowing en-bloc resection in lesions less than $2 \mathrm{~cm}$. Complete resection with conventional EMR can only be accomplished in a piecemeal fashion. This causes a major difficulty in assessment of the resection margins and carries the threat of incomplete resection and may cause a potential and high-risk of tumor recurrence or overtreatment $[11,20]$.

\subsection{Endoscopic Submucosal Dissection}

ESD was developed in the late 90s to remove larger lesions in an en-bloc resection. ESD outperforms EMR by providing a better rate of complete resection and a lower risk of local recurrence. However, this technique needs special skills, the procedure takes a long time and the procedure has a slightly higher incidence of complications.

In ESD procedures the mucosa surrounding the lesion is marked with a coagulation system, the lesion is raised with injection of a solution (saline, epinephrine, glycerol mixtures with methylene blue or indigo-carmine) and finally the submucosal layer is dissected circumferentially with lateral movements using different devices. Recently a water-jet-assisted knife is used to set the coagulation marks around the lesion, for circumferential incision and for dissection.

Recently, ESD has evolved in endoscopic surgery. New tools like magnetic anchor-guides ESD, springs, multitask devices, and double endoscopic intraluminal surgery, have been developed for a better visualization of the submucosal layer to reduce some technical problems associated to ESD [21].

The key goal of ESD and EMR is to make an accurate histological diagnosis. Thus, specimens must be oriented immediately after removal at the endoscopy unit. The depth of tumor invasion, degree of differentiation, lymphatic or vascular involvement and the status of the margin of the resection should be carefully reported for a correct staging and therefore to determine curability.

\subsection{Indications for Endoscopic Resection of EGC}

Initial conventional criteria for endoscopic resection in EGC were risk of lymph node metastases, technical problems and possibility of en-bloc resection.

The Japanese Gastric Cancer Association published guidelines to provide endoscopists the rationale for an appropriate use of these endoscopic techniques in the resection or EGC. Lesions that would fit into these absolute indications include: Differentiated adenocarcinoma 
without ulcerative findings, intramucosal lesions with a diameter less than $2 \mathrm{~cm}$ and no lymph involvement or metastasis in CT. These early indications for endoscopic removal of gastric cancer have been modified and extended according to additional surgical criteria. Current indications are shown in Table 5 [22].

However there are several issues to consider with extended indications which are the possibility of lymph node metastasis, overall in undifferentiated lesions, the histological discrepancy before and after resection of gastric adenoma and EGC, the different concept of gastric adenoma, dysplasia and adenocarcinoma in East and West and the possibility of histological mixed pattern in large lesions. Confirmation of no difference in the longterm survival between endoscopic treatment and conventional surgery is necessary in prospective studies.

\subsection{Management of Complications}

The rate of complications in endoscopic procedures for EGC resection varies among the different studies. The most common major complications are bleeding and perforation and some other complications may occur such as aspiration pneumonia, persistent pain and strictures.

The most common complication is bleeding; it is inevitable during the procedure and the most important thing is how to control bleeding effectively and reduce the bleeding rate. Clinically relevant bleeding occurs in up to $7 \%$ of patients undergoing these procedures. Early bleeding is more frequent in resections of tumors located in the upper third of the stomach because of greater vascularization. It can be stopped grasping the vessels with hot biopsy forceps, without stopping the procedure. Delayed bleeding is defined as hematemesis or melena at 0 to 30 days after the procedure and is strongly related to tumor location, size (mainly if $>40 \mathrm{~mm}$ ), recurrent lesions, and flat type [23]. In a metanalyses of 5 studies no statistical difference was seen on bleeding rates between the two procedures (OR 1.49; 95\%CI, 0.60 - 3.71) although a great heterogeneity was observed among the studies [24].

Perforation rate is relatively low and it seems to be higher with ESD than with EMR with an odds ratio of 4.67; 95\%CI, $2.77-7.87$ [24]. The risk of perforation during ESD is about $1 \%$ to $4 \%$. Perforation is also related to tumor location and ulceration but not to tumor size. Depending on the size, perforations can be frank or micro-perforation and according to the time of presentation immediate or delayed perforations. Perforation can be usually managed in a conservative manner using endoclips, fasting, broad-spectrum antibiotics and a nasogastric tube for few days. If a severe pneumoperitoneum appears, urgent decompression should be done to prevent life-threatening events like breathing disorders and/or neurogenic shock. The use of submucosal injection of polyethylene glicol or sodium hyaluronate has become popular in the last years to reduce perforation rates in ESD [23]. In a metanalyses including 8 studies, the perforation rate in a was higher in the ESD group (4.3\%) than in the EMR group (0.95\%) (OR 4.67; 95\%CI, 2.77 - 7.87) [24].

Minor complications that occur with ESD are pain, stricture and aspiration pneumonia. Pain after these procedures are usually well controlled by proton pump inhibitors (PPIs) and opioids. PPIs are also administrated for 8 weeks to prevent delayed bleeding complication and promote ulcer healing [25]. Strictures usually occur at the cardia and pylorus and they can be successfully treated by balloon dilatation [26]. Aspiration can be effectively prevented by frequent removal of gastric fluids and trying to avoid over-distention.

Little is known about mortality related to the procedures; it seems to be lower in the ESD than in EMR but the difference might not be statistically significant [24].

\section{Summary and Conclusion}

Early gastric incidence is increasing during the last years worldwide. It is believed that screening endoscopy could be a major factor in such increase. Endoscopic resection is one of the greatest advances in EGC treatment. It has become the standard treatment in most of the cases because early gastric cancer is associated with a low rate of lymph node metastasis and a high survival rate. Endoscopic Mucosal Resection (EMR) and more recently Endoscopic Submucosal Dissection (ESD) are the two main developed procedures. ESD achieves a higher rate of en-bloc resection, complete resection, curative resection and lower local recurrence compared with EMR group. The disadvantages associated with ESD, such as

Table 5. Treatment options in early gastric cancer.

\begin{tabular}{|c|c|c|c|c|c|c|}
\hline \multirow[b]{3}{*}{ Size $(\mathrm{mm})$} & \multicolumn{4}{|c|}{ Mucosal cancer } & \multicolumn{2}{|c|}{ Submucosal cancer } \\
\hline & \multicolumn{2}{|c|}{ No ulcer } & \multicolumn{2}{|c|}{ Ulcer present } & \multirow{2}{*}{$\frac{\mathrm{Sm} 1(<500 \mu)}{<30}$} & \multirow{2}{*}{$\frac{\operatorname{Sm} 2(>500 \mu)}{\text { Anysize }}$} \\
\hline & $<20$ & $>20$ & $<30$ & $>30$ & & \\
\hline Differentiated cancer & EMR & ESD & ESD & Surgery & ESD & Surgery \\
\hline Undifferentiated cancer & Surgery considered & Surgery & Surgery & Surgery & Surgery & Surgery \\
\hline
\end{tabular}

Modified from Gotoda, et al. [22]. 
higher perforation rates and longer procedure time, will probably improve as the endoscopists experience increases and new endoscopic tools are developed.

\section{REFERENCES}

[1] V. Baptista, A. Singh and W. Wassef, "Early Gastric Cancer: An Update on Endoscopic Management," Current Opinion in Gastroenterology, Vol. 28, No. 6, 2012, pp. 629-635. doi:10.1097/MOG.0b013e328358e5b5

[2] M. Tada, M. Shimada, F. Murakami, M. Mizumachi, K. Arima, H. Yanai, et al., "Development of Strip-Off Biopsy (in Japanese with English Abstract)," Gastrointestinal Endoscopy, Vol. 26, 1984, pp. 833-839.

[3] M. Hirao, K. Masuda, T. Asanuma, H. Nala, K. Noda, K. Matsuura, O. Yamaguchi and N. Ueda, "Endoscopicresection of Earlygastriccancerwith Local Injection of Hypertonicsaline-Epinephrine," Gastrointestinal Endoscopy, Vol. 34, No. 3, 1998, pp. 264-269. doi:10.1016/S0016-5107(88)71327-9

[4] K. Hosokawa and S. Yoshida, "Recentadvances in Endoscopic Mucosal Resection for Early Gastric Cancer (with English Abstract)," Japanese Journal of Cancer and Chemotherapy, Vol. 25, 1998, p. 483.

[5] N. Yamaguchi, H. Isomoto, E. Fukuda, K. Ikeda, H. Nishiyama, M. Akiyama, E. Ozawa, K. Ohnita, T. Hayashi, K. Nakao, S. Kohno and S. Shikuwa, "Clinical Outcomes of Endoscopic Submucosaldissection for Early Gastric Cancer by Indication Criteria," Digestion, Vol. 80, No. 3, 2009, pp. 173-181. doi:10.1159/000215388

[6] Japanese Gastric Cancer Association, "Japanese Classification of Gastric Carcinoma," Gastric Cancer, Vol. 1, 1998, pp. 10-24.

[7] Y. Ezoe, M. Muto, N. Uedo, H. Doyama, K. Yao, I. Oda, K. Kaneko, Y. Kawahara, C. Yokoi, Y. Sugiura, H. Ishikawa, Y. Takeuchi, Y. Kaneko and Y. Saito, "Magnifying Narrow Band Imaging Is more Accurate than Conventional White-Light Imaging in Diagnosis of Gastric Mucosal Cancer," Gastroenterology, Vol. 141, No. 6, 2011, pp. 2017-2025. doi:10.1053/j.gastro.2011.08.007

[8] J. Zhang, S. B. Guo and Z. J. Duan, "Application of Magnifying Narrow-Band Imaging Endoscopy for Diagnosis of Early Gastric Cancer and Precancerous Lesion," BMC Gastroenterology, Vol. 11, 2011, pp. 135-141. doi:10.1186/1471-230X-11-135

[9] P. Pimentel-Nunes, M. Dinis-Ribeiro, J. B. Soares, et al., "A Multicenter Validation of an Endoscopic Classification with Narrow Band Imaging for Gastric Precancerous and Cancerous Lesions," Endoscopy, Vol. 44, No. 3, 2012, pp. 236-246. doi:10.1055/s-0031-1291537

[10] R. M. Kwee and T. C. Kwee, "Imaging in Local Staging of Gastric Cancer: A Systematic Review," Journal of Clinical Oncology, Vol. 25, No. 15, 2007, pp. 2107-2116. doi:10.1200/JCO.2006.09.5224

[11] R. Seevaratnam, R. Cardoso, C. McGregor, L. Lourenco, A. Mahar, R. Sutradhar, C. Law, L. Paszat and N. Coburn, "How Useful Is Preoperative Imaging for Tumor, Node,
Metastasis (TNM) Staging of Gastric Cancer? A MetaAnalysis," Gastric Cancer, Vol. 15, Suppl 1, 2012, pp. 318. doi:10.1007/s10120-011-0069-6

[12] J. J. Bergman and P. Fockens, "Endoscopic Ultrasonography in Patients with Gastro-Esophageal Cancer," European Journal of Ultrasound, Vol. 10, No. 2-3, 1999, pp. 127-138. doi:10.1016/S0929-8266(99)00055-5

[13] S. Yamamoto, T. Nishida, M. Kato, T. Inoue, Y. Hayashi, J. Kondo, T. Akasaka, T. Yamada, S. Shinzaki, H. Iijima, M. Tsujii and T. Takehara, "Evaluation of Endoscopic Ultrasound Image Quality Is Necessary in Endosonographic Assessment of Early Gastric Cancer Invasion Depth," Gastroenterology Research and Practice, Vol. 2012, 2012, Article ID: 194530.

[14] S. Mocellin, A. Marchet and D. Nitti, "EUS for the Staging of Gastric Cancer: A Meta-Analysis," Gastrointestinal Endoscopy, Vol. 73, No. 6, 2011, pp. 1122-1134. doi:10.1016/j.gie.2011.01.030

[15] H. Yanai, T. Noguchi, S. Mizumachi, H. Tokiyama, H. Nakamura, M. Tada and K. Okita, "A Blind Comparison of the Effectiveness of Endoscopic Ultrasonography and Endoscopy in Staging Early Gastric Cancer," Gut, Vol. 44, No. 3, 1999, pp. 361-365. doi:10.1136/gut.44.3.361

[16] R. M. Kwee and T. C. Kwee, "Imaging in Local Staging of Gastriccancer: A Systematicreview," Journal of Clinical Oncology, Vol. 25, No. 15, 2007, pp. 2107-2116. doi:10.1200/JCO.2006.09.5224

[17] S. Bhandari, C. S. Shim, J. H. Kim, I. S. Jung, J. Y. Cho, J. S. Lee, M. S. Lee and B. S. Kim, "Usefulness of ThreeDimensional, Multidetector Row CT (Virtual Gastroscopy and Multiplanar Reconstruction) in the Evaluation of Gastric Cancer: A Comparison with Convention Alendoscopy, EUS, and Histopathology," Gastrointestinal Endoscopy, Vol. 59, No. 6, 2004, pp. 619-626. doi:10.1016/S0016-5107(04)00169-5

[18] M. Tanaka, H. Ono, N. Hasuike and K. Takizawa, "Endoscopic Submucosal Dissection of Early Gastric Cancer," Digestion, Vol. 77, Suppl. 1, 2008, pp. 23-28. doi:10.1159/000111484

[19] Y. M. Park, E. Cho, H. Y. Kang and J. M. Kim, "The Effectiveness and Safety of Endoscopic Submucosal Dissection Compared with Endoscopic Mucosal Resection for Early Gastric Cancer: A Systematic Review and MetaAnalysis," Surgical Endoscopy, Vol. 25, No. 8, 2011, pp. 2666-2677. doi:10.1007/s00464-011-1627-z

[20] T. Watanabe, K. Kume, M. Taip, M. Shibata, H. Kubo, Y. Ejiri and M. Otsuki, "Gastric Mucosal Cancer Smaller than $7 \mathrm{~mm}$ Can Be Treated with Conventional Endoscopic Mucosal Resection as Effectively as with Endoscopic Submucosal Dissection," Hepatogastroenterology, Vol. 57, No. 99-100, 2010, pp. 668-673.

[21] K. J. Kang, K. M. Kim, B. H. Min, J. H. Lee and J. J. Kim, "Endoscopic Submucosal Dissection of Early Gastric Cancer," Gut and Liver, Vol. 5, No. 4, 2011, pp. 418-426.

[22] T. Gotoda, "Endoscopic Resection of Early Gastric Cancer," Gastric Cancer, Vol. 10, No. 1, 2007, pp. 1-11. doi:10.1007/s10120-006-0408-1

[23] T. Gotoda, H. Yamamoto and R. M. Soetikno, "Endos- 
copic Submucosal Dissection of Early Gastric Cancer," Journal of Gastroenterology, Vol. 41, No. 10, 2006, pp. 929-942. doi:10.1007/s00535-006-1954-3

[24] J. Lian, S. Chen, Y. Zhang and F. Qiu, "A Meta-Analysis of Endoscopic Submucosal Dissection and EMR for Early Gastric Cancer," Testinal Endoscopy, Vol. 76, No. 4, 2012, pp. 763-770. doi:10.1016/j.gie.2012.06.014

[25] N. Uedo, Y. Takeuchi, T. Yamada, R. Ishihara, H. Ogiyama, S. Yamamoto, M. Kato, K. Tatsumi, E. Masuda, C. Tamai, S. Yamamoto, K. Higashino, H. Iishi and M. Tatsuta, "Effect of a Proton Pump Inhibitor or an H2Receptor Antagoniston Prevention of Bleeding from
Ulcer after Endoscopic Submucosal Dissection of Early Gastric Cancer: A Prospective Randomized Controlled Trial," The American Journal of Gastroenterology, Vol. 102, No. 8, 2007, pp. 1610-1616. doi:10.1111/j.1572-0241.2007.01197.x

[26] S. Coda, I. Oda, T. Gotoda, C. Yokoi, T. Kikuchi and H. Ono, "Risk Factors for Cardiac and Pyloric Stenosis after Endoscopic Submucosal Dissection, and Efficacy of Endoscopic Balloon Dilation Treatment," Endoscopy, Vol. 41, No. 5, 2009, pp. 421-426. doi: $10.1055 / \mathrm{s}-0029-1214642$ 